\title{
Normalization of Hypertension and Proteinuria in a Singleton Gestation; a Case Report and Literature Review
}

\author{
Bersaoui Marianne $^{1,2}$, Halimeh Rawad ${ }^{1,2, *}$, Msallem Mark ${ }^{2}$, Chedid Julien ${ }^{1,2}$, Feghali Joe ${ }^{1,2}$ \\ ${ }^{1}$ Department of Obstetrics and Gynecology, Saint George Hospital University Medical Center, Beirut, Lebanon \\ ${ }^{2}$ Faculty of Medicine, University of Balamand, Beirut, Lebanon
}

Email address:

rawad.halimeh@gmail.com (H. Rawad)

${ }^{*}$ Corresponding author

\section{To cite this article:}

Bersaoui Marianne, Halimeh Rawad, Msallem Mark, Chedid Julien, Feghali Joe. Normalization of Hypertension and Proteinuria in a Singleton Gestation; a Case Report and Literature Review. Journal of Gynecology and Obstetrics. Vol. 9, No. 1, 2021, pp. 26-30. doi: 10.11648/j.jgo.20210901.16

Received: February 10, 2021; Accepted: February 20, 2021; Published: February 27, 2021

\begin{abstract}
Preeclampsia is one of the most common causes of maternal mortality in the developed world. Until today, it is still one of the most researched entities in obstetrics mainly because it increases the risk of gestational complications, life-long sequalae and increased maternal and neonatal morbidity. Therefore, all causes of hypertension and /or proteinuria need to be ruled out of our differential diagnosis to get a clear definition of preeclampsia and manage it appropriately. Our case describes a patient with hypertension and proteinuria that presented after the $20^{\text {th }}$ week of gestation. In hospital management and close follow up was done with patient's symptoms, blood pressure, laboratory values and proteinuria resolving during her gestation leading to a good obstetrical and neonatal outcome. It has never been reported in literature to have a resolving case of hypertension and proteinuria in a singleton gestation resulting in a healthy living neonate. Our findings raise a lot of questions about the pathophysiology of the disease and whether preeclampsia can normalize before the delivery of the placenta. Therefore, is it time we start thinking about the maternal cardiovascular origin of preeclampsia instead of the placental vascular origin. We definitely need bigger studies to ascertain these clinical findings.
\end{abstract}

Keywords: Preeclampsia, Hypertension, Gestation, Pregnancy, Proteinuria

\section{Introduction}

Hypertension is notably one of the most common complications that can affect up to $10 \%$ of pregnancies worldwide. It is defined by either a systolic blood pressure of $140 \mathrm{mmHg}$ or higher and or a diastolic blood pressure of 90 $\mathrm{mmHg}$ or higher or both [1]. It increases the risk of gestational complications as well as life-long sequelae affecting the mother and fetus as they increase morbidity and mortality for both parties [2]. Hypertensive disorders present as one of the top 6 causes that increase maternal mortality and are implicated in at least $10 \%$ of maternal deaths [3].

The most common hypertensive disorders associated with pregnancy include "Chronic Hypertension" that precedes the onset of gestation or before the $20^{\text {th }}$ week of gestation and persists after delivery. On the other hand, "Gestational Hypertension" presents as elevated blood pressure after the $20^{\text {th }}$ week of gestation without proteinuria or signs of end organ dysfunction that resolves after delivery [1]. Finally, "Preeclampsia/Eclampsia" syndrome which remains one of the most common causes of maternal mortality in the developed world $[4,5]$.

Although the current literature shows evidence of preeclampsia resolution following fetal death in multifetal gestations, an extensive research revealed no reported cases of antenatal resolution of hypertension and proteinuria in a singleton gestation [6].

\section{Case Presentation}

We describe the case of a 29-year old Lebanese lady, primigravida (spontaneous pregnancy) presenting to the delivery unit at 26 weeks and 4 days of gestation for investigations and management of short cervix $(C L=17 \mathrm{~mm}$ in clinics on transvaginal ultrasound) and preterm contractions. 
On History, the patient reports hypertensive episodes during pregnancy (after 20 weeks of gestational age) reaching a maximum of $140 / 80 \mathrm{mmHg}$, not associated with any symptoms. Past medical and surgical histories were unremarkable. Patient denied cigarette smoking, alcohol intake, or any exposure to illicit drugs. She did not have any known food or drug allergies. The patient was started on vaginal progesterone (generic $200 \mathrm{mg}$ ) once per day.

Upon presentation, the patient had a blood pressure of $130 / 80 \mathrm{mmHg}$, heart rate of 80 beats per minute, and a temperature of 36.8 degrees Celsius. General physical examination was normal; digital cervical exam was not done. Patient was put on fetal monitoring which showed a reactive fetal heart rate, with irregular contractions felt by the patient.

Laboratory workup showed a complete blood count which was within normal ranges: WBC 9,700/mm3, Hemoglobin 12 g/dL, Platelets 135,000/mm3; SGPT 39 U/L, GGT 11 U/L, SGOT $26 \mathrm{U} / \mathrm{L}$, Lipase $41 \mathrm{U} / \mathrm{L}$, Direct Bilirubin $0.12 \mathrm{mg} / \mathrm{dL}$, Total Bilirubin $0.28 \mathrm{mg} / \mathrm{dL}$; PT-INR 0.98, PTT 23.88 seconds; C-Reactive Protein $0.34 \mathrm{mg} / \mathrm{L}$, creatinine $0.52 \mathrm{mg} / \mathrm{dL}$, Urea $20 \mathrm{mg} / \mathrm{dL}$; Urine analysis: RBC $1 / \mathrm{mm} 3$, WBC $50 / \mathrm{mm} 3$, Ketones negative and Proteins negative. Urine culture showed no growth after 48 hours of incubation. Vaginal culture showed normal vaginal flora. Obstetrical ultrasound showed a closed but short cervix measuring $13 \mathrm{~mm}$.

Once admitted, the patient was given Indomethacin $100 \mathrm{mg}$ for her preterm contractions then was switched to Calcium Chanel Blockers (Nifedipine 15mg every 8 hours) for tocolysis. A full course of dexamethasone for fetal lung maturity was given in the meantime due to the increased risk of preterm labor.

At 27 weeks and 4 days, the patient was still having irregular contractions on the monitor; a cervical pessary was inserted.

During her stay, blood pressure was being monitored and found to be high on many occasions reaching 140/90 $\mathrm{mmHg}$ although the patient was on Calcium Chanel blockers for tocolysis and dexamethasone administration for fetal lung maturity. Nifedipine was stopped to get true BP values, and pregnancy induced hypertension (PIH) work up was ordered.

Laboratory work up done at the time showed Urine spot ratio of 0.8 (higher than 0.3 ), platelets $128,000 / \mathrm{mm} 3$, stable liver function tests and 24 hour proteins in urine of $325 \mathrm{mg}$ (higher than $300 \mathrm{mg} / 24 \mathrm{hr}$ ), along with a BP value of $140 / 90 \mathrm{mmHg}$, hence the patient was started on magnesium sulfate for seizure prophylaxis since the patient met the criteria of preeclampsia.

Laboratory work up was then repeated every other day and showed a decrease in platelet levels reaching a nadir of $105,000 / \mathrm{mm} 3$ with stable hemoglobin levels and leukocyte counts. LFTs were also measured and showed an increase in SGOT levels reaching $125 \mathrm{U} / \mathrm{L}$ and SGPT levels reaching 68 $\mathrm{U} / \mathrm{L}$ on day 10 after presentation. Peripheral blood smear was ordered to assess thrombocytopenia and showed anisocytosis along with giant platelets.

Due to the disturbed LFTs, gastroenterology consult was requested, which prompted further investigations into a hepatic assessment including an abdominal ultrasound and daily follow-up on LFTs, PT/PTT, and serum protein levels. Abdominal ultrasound performed at the time showed a homogeneous liver which was normal in size along with a normal gallbladder, common bile duct, pancreas, and spleen. The kidneys were also normal in size with mild pelvicalyceal dilatation on the right side. PT, PTT, and serum protein levels were stable throughout her stay.

In the following days, daily 24-hour protein levels in were still constantly $>300 \mathrm{mg} / \mathrm{dL}$, with the highest value being $385 \mathrm{~g} / 24 \mathrm{~h}$. Hence, nephrology consult was requested for the assessment of proteinuria. In searching for differential diagnoses other than preeclampsia, glomerulonephritis had to be ruled out as a cause of proteinuria. Accordingly, additional laboratory tests were ordered, including serum ANA, C3, and $\mathrm{C} 4$ levels. ANA was negative at $1 / 80$, and $\mathrm{C} 3$ as well as $\mathrm{C} 4$ levels were normal at $161 \mathrm{mg} / \mathrm{dL}$ and $50 \mathrm{mg} / \mathrm{dL}$ respectively. No electrolyte abnormalities were detected during this time. In addition, serum creatinine was stable with a minimum value of $0.48 \mathrm{mg} / \mathrm{dL}$ and a maximum of $0.54 \mathrm{mg} / \mathrm{dL}$, reflecting a normal eGFR of $127-134 \mathrm{ml} / \mathrm{min} / 1.73 \mathrm{~m}^{2}$.

The patient's blood pressure was also being measured multiple times per day and reached a maximum of 160/100; she also started experiencing a headache but denied blurry vision. On physical exam, the patient did not have any pitting edema or lung auscultation abnormalities.

However, on day 12 of admission, the number of platelets started showing an increasing trend, eventually reaching $138,000 / \mathrm{mm} 3$ on day 30 of admission. SGOT levels which had reached a high of $125 \mathrm{U} / \mathrm{L}$ and SGPT levels which had reached a high of $68 \mathrm{U} / \mathrm{L}$, also began to show a decreasing trend, reaching a low of 17 and 18 , respectively by day 30 after presentation. Furthermore, $24 \mathrm{~h}$ urine protein collection which had reached a high of $385 \mathrm{~g} / 24 \mathrm{~h}$ began to decrease, eventually reaching $116 \mathrm{~g} / 24 \mathrm{~h}$ by the $30^{\text {th }}$ day after presentation. Blood pressure readings which were taken every 4 hours stabilized at a maximum of $130 / 80 \mathrm{mmHg}$ afterwards.

Throughout her stay, the patient was undergoing frequent monitoring via non-stress test, which showed a category 1 monitor with few painless contractions. She was also receiving a weekly obstetrical ultrasound to assess cervical length and fetal growth. The last ultrasound done on the $25^{\text {th }}$ day after presentation showed a cervical length of $35 \mathrm{~mm}$, EFW of $1.7 \mathrm{~kg}\left(80^{\text {th }}\right.$ percentile $)$, normal AFI, normal umbilical artery Doppler, a vertex baby, and a high and anterior placenta.

The patient was discharged on paracetamol and spasmolytic, and asked to repeat CBC, SGOT, SGPT, and 24hour protein in urine as outpatient 2 days after discharge.

The remainder of her pregnancy was uneventful. She presented to our facility again with frank rupture of membranes at 34 weeks and 2 days. She received a rescue shot of dexamethasone and underwent augmentation of labor with Pitocin.

She had an uneventful normal vaginal delivery of a healthy living baby with Apgar score of 9 and 10 at 1 and 5 minutes 
respectively, with a weight of $2680 \mathrm{gr}$ and height of $50 \mathrm{~cm}$ and was discharged on day 2 after delivery.

\section{Discussion}

\subsection{Preeclampsia with/Without Severe Symptoms}

ACOG modified the definition of preeclampsia by eliminating the necessity of having proteinuria as a vital criterion for establishing an accurate diagnosis. In case of absence of proteinuria, preeclampsia is diagnosed by the new onset of hypertension (SBP higher or equal to $140 \mathrm{mmHg}$ and/or DBP higher or equal to $90 \mathrm{mmHg}$ ) with the new onset of any of the following: thrombocytopenia less than $100,000 /$ microliter, renal insufficiency with serum creatinine levels greater than $1.1 \mathrm{mg} / \mathrm{dL}$ or a doubling of the serum creatinine concentration in the absence of any other renal cause, impaired liver functions with elevated transaminases to twice their normal concentration, new onset pulmonary edema, or cerebral or visual symptoms $[2,7]$.

Preeclampsia is subdivided according to the time of initial onset of symptoms into early-onset and late-onset disease. The early-onset variant presents before 34 weeks of gestation whereas the late-onset variant presents at or after the $34^{\text {th }}$ week of gestation. Those two variants have different outcomes concerning the neonate and the fetus with a 10 -fold increase in risk of perinatal death observed among mothers with early-onset disease and a two-fold increase in that risk among mothers with late-onset disease. It can also be classified according to its severity into preeclampsia with or without severe symptoms [8].

Risk factors for preeclampsia have been thoroughly studied and major risk factors have been identified such as previous history of preeclampsia, chronic hypertension, pregestational diabetes mellitus, antiphospholipid syndrome and obesity. Some other risk factors include SLE, nulliparity, advanced maternal age $>40$, genetic susceptibility and rare risk factors such as carrying a trisomy 13 fetus and a family history of preeclampsia [5, 9].

The pathophysiology of preeclampsia starts early on in gestation and before the onset of symptoms where an abnormal trophoblastic invasion and an alteration in the compliance of the spiral arteries of the uterus lead to an impaired abnormal placentation and reduced placental perfusion which will precipitate a systemic inflammatory response due to oxidative stress causing the heterogeneous symptoms of preeclampsia later on [10].

Preeclampsia is better managed when treatment includes three categories: prevention, early detection, and actual treatment. Prevention starts by identifying modifiable risks factors in women and optimizing their comorbid conditions such as encouraging smoking cessation, diet advice and changing their medications to new non-teratogenic ones to accommodate their preexisting diseases. According to the ASPRE trial, if a woman is considered to be at high risk for developing preeclampsia based on an algorithm that combines maternal factors, mean arterial pressure, uterine artery pulsatility index and maternal serum pregnancyassociated plasma protein-A and placental growth factor at 11 to $13^{\text {th }}$ week, she should be started on aspirin $(150 \mathrm{mg} \mathrm{qd})$ from the $11^{\text {th }}$ to $14^{\text {th }}$ weeks of gestations until the $36^{\text {th }}$ week of gestation which results in a $62 \%$ reduction in the risk of developing preterm preeclampsia [11]. Although there isn't a clear antenatal algorithm for the care for high risk women, they are seen more frequently antenatally and follow ups are more vigilant. Controlling the hypertension is crucial for the prevention of strokes especially in women with severe preeclampsia with a blood pressure equal to or greater than 160/100 mmHg. Antihypertensive medication that are pregnancy-safe (PO methyldopa, labetalol and calcium antagonists such as nifedipine) with labetalol being the firstline treatment should be carefully titrated in order to avoid a sudden drop in the mother's blood pressure compromising the uteroplacental circulation and causing fetal distress. If PO medication fail, IV bolus should be considered as the next line attempt at blood pressure normalization. Second-line treatment is hydralazine which is a smooth muscle relaxant. Finally, continuous fetal monitoring is essential in the management of severe disease [9].

HELLP (Hemolysis, Elevated Liver enzymes, Low Platelets) syndrome is a life-threatening form of preeclampsia which is caused by the activation of intravascular coagulation with fibrin deposition as a result of endothelial dysfunction causing the classical triad of hemolysis which can be sensitively monitored by haptoglobin, elevated AST and ALT levels and thrombocytopenia with a characteristic RUQ or epigastric pain associated with nausea, vomiting and malaise in up to $30 \%$ of the cases. There is a consensus that a HELLPcomplicated pregnancy between 32-34 weeks should be immediately delivered [12].

The definitive cure of preeclampsia is fetal delivery and should only be considered in the absence of fetal distress and after stabilization of the mother hemodynamically, neurologically and biochemically [13].

Magnesium sulfate $\left(\mathrm{MgSO}_{4}\right)$ is vital in treatment of severe preeclampsia. It acts in two ways: the first being halting the progression into eclampsia which is characterized by preeclampsia with the new onset of convulsions, and the second being blunting eclamptic convulsions. It is given intravenously and patients should be closely monitored since this treatment has severe adverse toxicity effects which are paralysis, absence of reflexes, respiratory drive depression, and arrhythmias. In case of toxicity, stop the treatment and give calcium gluconate [9].

After a thorough search of the medical literature, no reported cases of improvement of symptoms of preeclampsia with normalization of lab values to back to their original baseline without treatment have been identified in a singleton gestation.

\subsection{Proteinuria Differential in a Pregnant Woman}

Physiologically, pregnancy demonstrates an increase in effective renal plasma flow up to $75 \%$ of non-pregnant 
baseline by 16 weeks of gestation up to 34 weeks of gestation and by the end of the first trimester, GFR increases up to $50 \%$ of its non-pregnant baseline (150-200 $\mathrm{mL} / \mathrm{min}$ compared to $120 \mathrm{~mL} / \mathrm{min}$ ). This physiological increase corresponds to an increase in urinary protein excretion from $60-90 \mathrm{mg} / 24 \mathrm{~h}$ to $180-250 \mathrm{mg} / 24 \mathrm{~h}$ but this increase shouldn't reach proteinuria levels [14, 15]. Even though laboratory signs of proteinuria in pregnancy with gestational hypertension is highly suspicious of preeclampsia and the patient should be worked up and closely monitored, other causes of proteinuria need to be ruled out.

Proteinuria is defined as greater than $300 \mathrm{mg}$ of urinary protein excretion in a $24 \mathrm{~h}$ urine collection, a protein-tocreatinine ratio greater than $300 \mathrm{mg}$ or persistent proteinuria (greater or equal $1+$ on dipstick on at least two occasions at least 4 hours apart). Proteinuria in pregnancy is classified into proteinuria before or after the $20^{\text {th }}$ week of gestation. Evaluation of the onset of proteinuria is crucial for diagnosis. In patients who are previously diagnosed with chronic renal disease or those who present with an onset of proteinuria before the $20^{\text {th }}$ week of gestation, a diagnosis of renal disease can be established. On the other hand, new onset proteinuria after the $20^{\text {th }}$ week of gestation is usually the first presenting sign of preeclampsia $[16,17]$.

\subsubsection{Pre-gestational Diabetes}

Diabetes Mellitus is one of the most common disorders in pregnancy and could cause proteinuria through glomerular damage [18]. 2-5\% of pregnant women with Pre-gestational Diabetes Mellitus are at risk of developing nephropathy putting them at a high risk of developing preeclampsia [19]. The patient discussed in this case report had a normal glucose profile and the suspicion of any presentation of Diabetes Mellitus was very low.

\subsubsection{Chronic Hypertension}

Chronic hypertension could also cause proteinuria in a pregnant woman. It is fairly easy to diagnose chronic hypertension in a primigravida who is being monitored by her obstetrician because by definition it is the onset of hypertension before the $20^{\text {th }}$ week $[18,19]$. Our patient didn't have hypertension before her $20^{\text {th }}$ week of gestation so she couldn't have developed a superimposed preeclampsia.

\subsubsection{Renal Causes of Proteinuria}

Other causes of proteinuria in pregnancy include chronic kidney diseases. Chronic kidney disease can cause proteinuria but present before pregnancy or early in pregnancy usually before the $20^{\text {th }}$ week of gestation $[16,17]$. Our patient had no onset of proteinuria before the $20^{\text {th }}$ week of gestation therefore excluding most of the renal causes of proteinuria. Glomerular disease remains the biggest entity for evaluation which mainly includes glomerulonephritis, nephrotic syndrome, and non nephrotic glomerular proteinuria. Our patient had a maximal proteinuria of $385 \mathrm{mg} /$ day (not exceeding $500 \mathrm{mg} /$ day), had no declining kidney function (good eGFR) and no evidence of systematic disorder therefore excluding most causes of glomerulonephritis. Nephrotic syndrome also ruled out in our case. Patient had no peripheral edema, no hypoalbuminemia (serum albumin nadir of $3.2 \mathrm{~g} / \mathrm{l}$ ) and no hyperlipidemia/hyperlipiduria.

Infectious etiologies in the urinary tract can also cause proteinuria in a pregnant woman. With the increase in size of the gravid uterus, mechanical compression on the urinary system can cause stasis and increase the patient's risk of developing an asymptomatic bacteriuria which can progress into a more serious pyelonephritis. Pyelonephritis should be treated aggressively and is more likely to be associated with azotemia [18]. However, our patient didn't have azotemia on her electrolyte differential, nor a fever or elevated inflammatory markers suggestive of an infectious etiology. Furthermore, her urine analysis and culture came back negative for microorganisms.

Urinary tract obstruction through the means of a kidney stone could also cause proteinuria in a pregnant woman. Even though the dilation of the ureteropelvic system is present when there is an obstruction in the urinary tract, pregnancy is thought to have the same dilatory effect on the urinary collecting system through the hormonal effect of increased progesterone and the mechanical obstruction of the growing uterus, giving the wrong impression of an obstructive uropathy $[18,20]$. Our patient had no urinary tract obstruction documented by ultrasound.

Lupus nephritis is an immune-complex glomerulonephritis that precipitates a nephritic syndrome in approximately $50 \%$ of SLE patients. The hallmark of Lupus nephritis is nephritic range proteinuria and autoantibodies. Those immune complexes are made mostly of $\operatorname{IgG}, \operatorname{IgA}$, IgM as well as C3, C4 and C1q [21]. The patient discussed didn't have a prior diagnosis of SLE or any form of suspicion and if her first manifestation of the disease was to be nephritis, it is highly unlikely and rare for that to happen according to Arbel $O$ et al (2005) [6]. Our patient didn't meet SLE criteria on history and physical exam. ANA titer and $\mathrm{C} 3$ and $\mathrm{C} 4$ complement levels were also measured and were found to be normal which makes the diagnosis of lupus nephritis highly unlikely.

\section{Conclusion}

Preeclampsia is still considered as one of the most common causes of maternal and neonatal mortality and morbidity. Therefore, a thorough clinical and laboratory workup should be done to exclude other pathological entities that present similarly. Our case presents the first normalization of hypertension and proteinuria in pregnancy prior to delivery of the placenta. It raises a lot of questions about the pathophysiology of this entity and whether preeclampsia can normalize before the delivery of the placenta. Therefore, is it time we start thinking about the maternal cardiovascular pathophysiology of preeclampsia instead of the placental vascular origin. 


\section{Conflicts of Interest}

The authors declare that they have no conflicts of interest.

\section{Funding}

This work was funded by Joe Feghali, MD.

\section{References}

[1] Markham K., and Funai E. F.: Pregnancy-related hypertension. In Creasy R. K., Resnik R., and Iams J. D. (eds): Creasy and Resnik's maternal-fetal medicine, 7th edition. Philadephia: Elsevier, 2015. pp. 756-781.

[2] American College of Obstetricians and Gynecologists. 2013. Hypertension in pregnancy. Report of the American College of Obstetricians and Gynecologists' Task Force on Hypertension in Pregnancy. Obstetrics and Gynecology 122: 1122-1131.

[3] Creanga A. A., Berg C. J., Syverson C., et al: Pregnancyrelated mortality in the United States, 2006-2010. Obstet Gynecol 2015; 125: pp. 5-12.

[4] Peter von Dadelszen, Laura A. Magee \& James M. Roberts (2003) Subclassification of Preeclampsia, Hypertension in Pregnancy, 22: 2, 143-148, DOI: 10.1081/PRG-120021060.

[5] Rana, S., Lemoine, E., Granger, J. P., \& Karumanchi, S. A. (2019). Preeclampsia. Circulation Research, 124 (7), 10941112. doi: 10.1161/circresaha.118.313276.

[6] Pham, A., Rosenthal, E., Roman, A., Makhamreh, M., Berghella, V., Farhi, F., \& Al-Kouatly, H. B. (2020). Preeclampsia resolution after fetal death in multifetal gestation: A systematic literature review. American Journal of Obstetrics and Gynecology, 222 (4), 385-389. doi: 10.1016/j.ajog.2019.10.097.

[7] (2013). Hypertension in Pregnancy. Obstetrics \& Gynecology, 122 (5), 1122-1131. doi: 10.1097/01.AOG.0000437382.03963.88.

[8] Lisonkova S, Joseph KS. (2013) Incidence of preeclampsia: risk factors and outcomes associated with early- versus lateonset disease. Am J Obstet Gyneco; 209: 544. e1-12.

[9] English, F. A., Kenny, L. C., \& McCarthy, F. P. (2015). Risk factors and effective management of preeclampsia. Integrated blood pressure control, 8, 7-12. doi: 10.2147/IBPC.S50641
[10] Lopez-Jaramillo, P., Barajas, J., Rueda-Quijano, S. M., LopezLopez, C., \& Felix, C. (2018). Obesity and Preeclampsia: Common Pathophysiological Mechanisms. Frontiers in Physiology, 9. doi: 10.3389/fphys.2018.01838.

[11] Rolnik D. L., Wright D., Poon L. C., et al. Aspirin versus placebo in pregnancies at high risk for preterm preeclampsia. The New England Journal of Medicine. 2017; 377 (7): 613622. doi: 10.1056/NEJMoa1704559.

[12] Rath, W., Faridi, A. \& Dudenhausen, J. (2005). HELLP Syndrome. Journal of Perinatal Medicine, 28 (4), pp. 249-260. Retrieved 9 Dec. 2019, from doi: 10.1515/JPM.2000.033.

[13] Uzan, J., Carbonnel, M., Piconne, O., Asmar, R., \& Ayoubi, J. M. (2011). Pre-eclampsia: pathophysiology, diagnosis, and management. Vascular health and risk management, 7, 467474. doi: 10.2147/VHRM.S20181.

[14] Airoldi, J. \& Weinstein, L. (2007). Clinical Significance of Proteinuria in Pregnancy. Obstetrical \& Gynecological Survey, 62 (2), 117-124. doi: 10.1097/01.ogx.0000253301.55009.ac.

[15] Sibai, B. (2021). Preeclampsia and Hypertensive Disorders. In M. Landon (Author), Gabbe's Obstetrics: Normal and Problem Pregnancies (7th ed., Vol. 38, pp. 708-750). Elsevier.

[16] Ekiz A, Kaya B, Polat I, et al. The outcome of pregnancy with new onset proteinuria without hypertension: retrospective observational study. J Matern Fetal Neonatal Med 2016; 29: 1765 .

[17] Yamada T, Obata-Yasuoka M, Hamada H, et al. Isolated gestational proteinuria preceding the diagnosis of preeclampsia - an observational study. Acta Obstet Gynecol Scand 2016; 95: 1048.

[18] Podymow T., August P., Ayub Akbari A.: Management of Renal Disease in Pregnancy, Obstetrics and Gynecology Clinics, 2010-06-01, Volume 37, Issue 2, Pages 195-210.

[19] Damm J. A., Asbjornsdottir B., Callesen N. F., et al: Diabetic nephropathy and microalbuminuria in pregnant women with type 1 and type 2 diabetes: prevalence, antihypertensive strategy, and pregnancy outcome. Diabetes Care 2013; 36: pp. 3489-3494.

[20] Ramin S. M., Vidaeff A. C., Yeomans E. R., et al: Chronic renal disease in pregnancy. Obstet Gynecol 2006; 108: pp. 1531-1539.

[21] Arbel $\mathrm{O}$ et al: Hyperacute renal failure as the initial presentation of systemic lupus erythematosus. Lupus. 14 (4): 331-3, 2005. 